\title{
Auditory Event-Related Potentials in Antipsychotic-Free Subjects With Ultra-High-Risk State and First-Episode Psychosis
}

\author{
Ming H. Hsieh 1,2,3, Yi-Ting Lin 1,2, Yi-Ling Chien 1,2, Tzung-Jeng Hwang 1,2,3, \\ Hai-Gwo Hwu ${ }^{1,2,3}$, Chih-Min Liu ${ }^{1,2,3 *}$ and Chen-Chung Liu ${ }^{1,2 *}$ \\ ${ }^{1}$ Department of Psychiatry, National Taiwan University Hospital and College of Medicine, Taipei, Taiwan, ${ }^{2}$ Department of \\ Psychiatry, College of Medicine, National Taiwan University, Taipei, Taiwan, ${ }^{3}$ Graduate Institute of Brain and Mind Sciences, \\ College of Medicine, National Taiwan University, Taipei, Taiwan
}

\section{OPEN ACCESS}

Edited by:

Yen Kuang Yang,

National Cheng Kung University

Hospital, Taiwan

Reviewed by:

Kao Chin Chen,

National Cheng Kung University,

Taiwan

Michael Kiang,

Centre for Addiction and Mental Health (CAMH), Canada

${ }^{*}$ Correspondence:

Chih-Min Liu

cmliu1968@ntu.edu.tw

Chen-Chung Liu

chchliu@ntu.edu.tw

Specialty section:

This article was submitted to Schizophrenia,

a section of the journal

Frontiers in Psychiatry

Received: 14 December 2018

Accepted: 26 March 2019

Published: 15 April 2019

Citation:

Hsieh $M H$, Lin $Y-T$, Chien $Y-L$, Hwang T-J, Hwu H-G, Liu C-M and

Liu C-C (2019) Auditory EventRelated Potentials in AntipsychoticFree Subjects With Ultra-High Risk State and First-Episode Psychosis.

Front. Psychiatry 10:223.

doi: 10.3389/fpsyt.2019.00223
Background: Auditory event-related potentials (ERPs) have been utilized to study defective information processing of patients with schizophrenia. To delineate the pathophysiological processes from pre-psychotic state to first-episode psychosis, a study on subjects from ultra-high-risk (UHR) state to first-episode psychosis, ideally in an antipsychotic-free condition, can add important information to our understanding.

Methods: Patients with UHR state or at their first-episode psychosis (FEP) who were drug-naive or only have been temporarily treated with antipsychotics were assessed by auditory ERPs measurement, including P50/N100 (sensory gating) and duration mismatch negativity (MMN; deviance detection). A group of age-matched healthy subjects served as their controls.

Results: A total of 42 patients (23 UHR and 19 FEP) and 120 control subjects were recruited, including 21 pure drug-naive and 21 with very short exposure to antipsychotics. Collapsing FEP and UHR as a patient group, they exhibited significant sensory deficits manifested as larger P50 S2 amplitude, larger N100 ratio, and smaller N100 difference, and significantly less deviance detection response revealed by MMN. Such differences were less significant when treating FEP and UHR separately for comparisons. Comparisons of ERP results between drug-naive subjects and antipsychotic-short-exposure subjects revealed no significant difference in any P50/N100 and MMN parameter.

Conclusion: Our study is one of the few studies focused on drug-naive or minimally treated patients at pre- or early-psychotic states. Our results exhibited impaired performance in sensory gating and deviance detection shown by certain parameters. A longitudinal study with larger sample sizes will be helpful to provide more evidence to elucidate the role of antipsychotics on an individual's neurophysiological performance at different stages of psychosis.

Keywords: event-related potentials, first-episode psychosis, mismatch negativity, N100, P50, schizophrenia, ultra-high risk 


\section{INTRODUCTION}

Neuroscience tools have been widely employed in schizophrenia research in recent decades (1-3). Neurobiological impairments precede the onset of a full clinical syndrome. Therefore, we can delineate psychopathological progresses by careful assessment throughout the pre-psychotic and early-psychotic states (4). Among the various neuroimaging methods, auditory eventrelated potentials (ERPs) have been utilized to study normal versus defective information processing of neuropsychiatric disorders, such as schizophrenia $(1,3,5)$. Successful processing of sensory inputs requires two kinds of ability: sensory gating, the ability to inhibit intrinsic responses to redundant stimuli, and deviance detection, the ability to facilitate responses to less frequent salient stimuli (6). Using ERP components as measuring instruments, P50/N100 suppression represents the extent of inhibitory failure (impaired sensory gating), while MMN (mismatch negativity) indicates the magnitude of impaired deviance detection. Both processes are thought to be "pre-attentive" (passive, not demanding on subject's active attention) and have been found to be impaired in patients with schizophrenia $(3,5,7)$. Evidence suggests that auditory P50, N100, and MMN could be candidate endophenotypes of schizophrenia with intermediate relationship to susceptible genes of schizophrenia $(3,6,8)$, serve as potential biomarkers to specify the progress of illness (9-12), and even help to predict if a subject would convert to full-blown psychosis (13).

As most neurobiological studies of schizophrenia were conducted in chronic patients, the possible negative impact brought by long duration of illness and long-term use of antipsychotics on brain neurochemistry and possibly on brain morphology (14) could be confounders and make it difficult to interpret those neurobiological findings (15-17). Similarly, even though P50 suppression and MMN has been regarded as endophenotypes for schizophrenia $(6,18,19)$, the findings of duration MMN deficits were absent in a few studies focused on subjects at their first-episode psychosis (FEP) $(7,20-23)$, as well as there are studies that failed to reveal P50/N100 sensory gating deficits in this population $(24,25)$. However, in the studies including first-episode psychosis, whether patients were drug-free, continuing antipsychotics, or temporarily holding off antipsychotics was not all well controlled during assessment of ERPs. Ignoring such a difference in medication status may lead to confusing results (26), while administration of antipsychotics have been shown to influence ERP results, although the direction and extent of impact were diverse in different antipsychotics (27-32).

To circumvent the impact of long duration of illness and use of antipsychotics, examining subjects with drug naivety is an ideal approach. In schizophrenia research, attention has been directed towards the early state or even "pre-psychotic" state of full-blown psychosis. A lot of studies have been focused on this critical period, not only for identifying factors predicting conversion to psychosis or how to modify the trajectories of psychosis (33), but also for disentangling the complex pathogenesis of schizophrenia-related psychosis (34). The ultra-high risk (UHR), also known as late prodrome, model depicting a group of subjects who had subthreshold psychotic symptoms yet not developed full-blown psychosis (35), has been transformed into an attenuated psychosis syndrome in the Diagnostic and Statistical Manual of Mental Disorders (DSM-5), Section III, as a category in need of more investigations (36). Furthermore, theoretically, Keshavan et al. pushed the model back to the beginning in the course of psychosis and named an early prodromal state with nonspecific symptoms and/or basic symptoms as the "early/broadly defined at-risk mental states" (E-BARS) (37) to capture all possible features that happened during the formation of psychosis.

Our research team has started a prospective study on the psychopathological progress of the pre-psychotic state (the SOPRES study) in 2006 (38). We have recruited subjects at a gradient of clinical severities spanning from the E-BARS to UHR and FEP, together with a group of normal controls. Our ERP results of this cohort revealed a gradient of P50/N100 sensory-gating deficits across different levels of clinical severity (likely a state marker), while impaired deviance detection exhibited by duration MMNs was already detectable in people at pre-psychotic states and not much different from that in FEP (likely a trait marker) (39). But like most previous studies, the SOPRES did not control a patient's medication status. In 2008, we initiated an open-label drug trial on UHR and first-episode psychosis, focused on those who were drug-naive or have only received a short period of antipsychotic treatment (40). The baseline assessment of this sample allows us to examine to what extent the auditory ERP components (P50/ MMN/N100) will be different between subjects with UHR state and patients at first-episode psychosis, spared from the influence of antipsychotics, and compared to a large group of healthy controls.

\section{METHODS}

\section{Subjects}

This study was approved by the Institutional Review Board of the National Taiwan University Hospital. Written informed consent was received from all participants, including written assent given by minors with informed consent from their parents. Subjects were those who participated in a 4-week open-label clinical trial using flexible dose of aripiprazole on patients with UHR state or at their first-episode psychosis between July 2008 and June 2016. Details of the clinical trial procedures have been addressed in our previous publication (40), and the definition of clinical cases is briefed below. The controls were recruited by responding to ads of various studies conducted by our schizophrenia research team with the prerequisite of having no lifetime or current psychiatric diagnosis or family history of psychotic disorders. Those who had a psychotic episode for more than 1 year, a mood episode, current use of psychoactive substance, a history of central nervous system illness or traumatic brain injury, an IQ below 70, and pregnancy were excluded from recruitment.

\section{Definition of Clinical Cases}

The FEP subjects were those who developed full-blown psychosis that met the Diagnostic and Statistical Manual of Mental Disorders, Fourth Edition (DSM-IV) criteria for schizophrenia or schizophreniform disorder within the recent 1 year. The UHR subjects presented subthreshold psychotic symptoms meeting the 
comprehensive assessment of at-risk mental status criteria (41) either with attenuated psychotic symptoms or with brief limited intermittent psychotic symptoms. Subjects have never received antipsychotic treatment before were designated as the "drug-naive" group. Subjects reported to have received a known antipsychotic or psychotropic agent that exerted an effect or adverse event very likely to be associated with antipsychotics for a total of less than 3 months were designated as the "antipsychotic-short-exposure" group.

The antipsychotic-short-exposure group was asked to remain antipsychotic-free for at least 1 week before baseline assessments. Patient's clinical severity was assessed by a Mandarin version of the Positive and Negative Syndrome Scale (PANSS) for schizophrenia and received ERP studies at baseline and 4 weeks after completing treatment with aripiprazole. In this paper, we focused on their baseline ERP results that were not affected by antipsychotic treatment.

\section{Testing Environment}

Before ERP recording, audiometry testing was used to exclude subjects who could not detect $40-\mathrm{dB}$ sound pressure level tones at $500,1,000$, and $6,000 \mathrm{~Hz}$ presented to either ear. The standard procedures for auditory $\mathrm{P} 50 / \mathrm{N} 100$ and MMN paradigm were based on established protocols (42-45). The participants had not smoked for at least $1 \mathrm{~h}$ before sessions (46) and were asked to lie down in supine position in a comfortable recliner in a soundattenuating, electrically shielded booth and instructed to relax with his/her eyes open and to focus on a fixation point (P50 and N100 session) or a cartoon running with no sound on the video monitor (MMN session). There were no tasks performed during the test. During the testing, electroencephalography and stimuli would be recorded continuously, and subjects were closely observed through a video monitor. They would be monitored visually and by electroencephalography (EEG) for signs of sleep or slow wave activity, which, if present, prompted the experimenter to speak briefly with the subject.

The EEG signals were recorded with a Quik-Cap (Compumedics Neuroscan, El Paso, TX, USA) from 32 scalp locations. According to the Quik-Cap website, all electrodes were placed according to the International 10-20 electrode placement standard. Electrodes placed at the tip of the nose and at Fpz served as the reference and ground, respectively. Four additional electrodes were located above and below the left eye and at the outer canthi of both eyes to monitor blinks and eye movements. All electrode impedances were kept below $5 \mathrm{k} \Omega$ prior to recording.

\section{Stimuli Session and ERP Recording}

The auditory stimuli were generated by a Neuroscan STIM system, and data were recorded on a Neuroscan ACQUIRE system (Compumedics Neuroscan, El Paso, TX, USA). Stimuli were digitized at a rate of $1 \mathrm{kHz}$, and an online band-pass filter at $0.5-100 \mathrm{~Hz}$, without $60-\mathrm{Hz}$ notch filter, was applied. Auditory stimuli were presented to the subjects binaurally via foam insert earphones in two consecutive sessions, i.e., the session of paired-click paradigm for P50/N100 followed by the duration MMN session.
Online averaging was used to monitor the number of trials free from gross artifacts (defined as activities exceeding $\pm 100 \mu \mathrm{V}$ in the -100 to $500 \mathrm{~ms}$ time window following stimuli). Regarding the paired-click P50/N100 paradigm, paired auditory clicks (1 ms, $85 \mathrm{~dB}$ ) were presented every 8-12 s through the whole test session (average: $10 \mathrm{~s}$ ), with a 500-ms interstimulus interval $(47,48)$. The paired-click P50/N100 session was terminated when a minimum of 120 artifact-free trials had been obtained, which took about $30 \mathrm{~min}$. For the duration MMN paradigm, pure tone stimuli $(1 \mathrm{kHz}, 85-\mathrm{dB}$ SPL, 5-ms rise/fall) were generated by the Neuroscan STIM system. The auditory stimuli consisted of standard stimuli (90\%, 50-ms duration) and deviant stimuli (10\%, 100-ms duration) delivered in a pseudo-random order with the constraint that deviant stimuli could not be repeated back to back. The cartoon soundtrack was turned off and replaced by the experimental auditory stimuli that were presented at a fixed 500-ms onset-to-onset asynchrony. The MMN session was continued until a minimum of 225 artifact-free deviant trials had been collected online, which took approximately $30 \mathrm{~min}$.

\section{Offline Data Processing}

Details regarding offline signal analysis, using Neuroscan Edit 4.5 software (Compumedics Neuroscan, El Paso, TX, USA), were followed as our previous publications $(39,44,49)$. All data were processed by researchers who were blind to the subject's group (50). Semi-automated procedures using the Tool Command batch processing language (TCL) began with electrooculography (EOG) artifact reduction through a built-in pattern-recognition algorithm (51). For P50/N100, the data were epoched for the time window from -100 to $923 \mathrm{~ms}$ of the first click, covering both S1 and S2 in the same epoch. All epochs containing activities exceeding $\pm 50 \mu \mathrm{V}$ were excluded. To prevent temporal aliasing, epochs were averaged and digitally band-pass-filtered ( 10 to $50 \mathrm{~Hz}$ for P50, 1 to $50 \mathrm{~Hz}$ for N100) in the frequency domain. Trials with artifacts were manually rejected. By using preset intervals, peaks and preceding troughs were then automatically detected at the $\mathrm{Cz}$ electrode. The P50 peak was defined as the largest positive deflection between 45- and 75-ms poststimulus, and its amplitude was assessed as the difference between this peak and the preceding negative trough (not earlier than $30-\mathrm{ms}$ poststimulus). The N100 component was identified as the most negative deflection within 80 - to 150 -ms poststimulus, and N100 amplitude was defined as the absolute difference between the N100 peak and the preceding positive trough. P50 and N100 parameters included the S1 amplitude, S2 amplitude, amplitude difference (S1 - S2), and P50/N100 gating ratio (S2/S1). A maximum gating ratio of 2 was used to prevent outliers from disproportionately affecting the group means $(39,44,52)$.

For duration MMN analysis, each subject's continuous data file to 500-ms poststimulus. EEG responses to standard and deviant stimuli were separately averaged to create a standard ERP and a deviant ERP, and both were low-pass filtered at $20 \mathrm{~Hz}$ (0-phase shift and 24-dB/octave roll-off) to remove any residual high-frequency artifacts. MMN waveforms were generated by subtracting the standard ERP from the deviant ERP. MMN indices were measured as the mean voltage from 135 to $205 \mathrm{~ms}$ of the Fz electrode $(18,39,53-55)$. 


\section{Statistical Analysis}

Statistical analyses were performed using SPSS v16.0 software (SPSS, Chicago, IL). For demographic characteristics and ERP parameters, the results are presented in means and standard deviations $( \pm \mathrm{SD})$. Chi-square tests were used for categorical variables. Putting subjects with UHR state and first-episode psychosis together as a patient group, we compare control vs. patient group in demographics and ERP results. In addition, comparison between controls, UHR, and FEP groups with analysis of variance (ANOVA) was performed, and we also calculated comparison between control/drug-naive/ antipsychotic-short-exposure groups. All post hoc comparisons were made using the Scheffe test. Statistical significance was set at $p<0.05$. Cohen's $d$ effect size was calculated for all ERP parameters.

\section{RESULTS}

A total of 42 patients (19 FEP and 23 UHR) and 120 control subjects were recruited. Among them, 21 patients endorsed pure drug naivety (7 FEP and $14 \mathrm{UHR}$ ), and the other 21 patients (12 FEP and 9 UHR) have only been exposed to antipsychotics for no longer than 3 months. Indeed, the majority of these 21 shortexposure patients took antipsychotics at a low dose level no longer than 4 weeks and they could endure a washout period of 1 week prior to receiving ERP assessment with no apparent worsening of symptoms. Both paired-click P50/N100 paradigm and duration MMN paradigm took about $30 \mathrm{~min}$ in duration. Although all 42 patients had ERP recorded, 9 patients (3 UHR/6 FEP) could not finish the P50/N100 paradigm, while 8 patients (5 UHR/3 FEP) could not tolerate duration MMN paradigm, yielding the numbers of subjects with data available for further analysis to be 33 and 34 for P50/N100 and MMN, respectively.

\section{Demographic and Clinical Characteristics}

In Table 1, UHR and FEP were treated collectively as a patient group to compare with the control group, while in Table 2, UHR and FEP were examined separately for any difference between these two groups. There were no statistical differences in age and gender when the patient group is compared to the control group, although the UHR group was significantly younger than the FEP group ( $23.64 \pm 5.08$ vs. $28.45 \pm 8.33, p=0.022)$. The controls had 1.6 years more in education and reported much lower amount of smoking compared to the patient group, while there was no difference in these two variables between the UHR and FEP groups. In terms of clinical severity shown by PANSS scores, the UHR patients only exhibited lower scores in positive symptom subscales than the FEP patients $(15.0 \pm 2.9$ vs. $19.4 \pm 4.6, p<$ 0.001 ), while their scores in negative symptoms and general symptoms subscales were comparable to each other.

\section{Comparisons of Event-Related Potentials}

Also presented in Table 1, the patient group had a smaller magnitude in MMN, a larger P50 S2 amplitude, a larger N100 amplitude ratio, and a smaller N100 difference compared to the control group. However, as detailed in Table 2, the patient

TABLE 1 | Demographics and ERP results of control and patient groups (SD in parentheses). ${ }^{a}$

\begin{tabular}{|c|c|c|c|c|}
\hline & Control & Patients & Statistics & Effect size (Cohen's $d$ ) \\
\hline & $n=120$ & $n=42$ & & \\
\hline Age & $26.63(5.09)$ & $25.82(7.08)$ & 0.424 & \\
\hline Male gender $(\%)^{b}$ & $63(52.5 \%)$ & $21(50 \%)$ & $\chi^{2}=0.08, p=0.78$ & \\
\hline Education (years) & $15.62(1.88)$ & $14.00(2.51)$ & $<0.001^{\star \star}$ & \\
\hline Smoking PPD & $0.03(0.1)$ & $0.15(0.4)$ & $0.035^{\star}$ & \\
\hline \multicolumn{5}{|l|}{ PANSS } \\
\hline Positive symptoms (P1 to P7) & - & $17.0(4.3)$ & & \\
\hline Negative symptoms (N1 to N7) & - & $14.1(5.9)$ & & \\
\hline General psychopathology (G1 to G16) & - & $35.3(8.6)$ & & \\
\hline MMN Fz & $-1.36(0.81)$ & $-1.05(0.78)$ & $0.047^{*}$ & 0.39 \\
\hline \multicolumn{5}{|l|}{ P50 } \\
\hline S1 amplitude & $2.44(1.06)$ & $2.53(1.4)$ & 0.679 & 0.07 \\
\hline S2 amplitude & $1.09(0.64)$ & $1.45(0.84)$ & $0.008^{\star \star}$ & 0.48 \\
\hline P50 ratio & $0.51(0.34)$ & $0.63(0.38)$ & 0.075 & 0.33 \\
\hline P50 difference & $1.35(1.07)$ & $1.08(1.39)$ & 0.239 & 0.22 \\
\hline \multicolumn{5}{|l|}{ N100 } \\
\hline S1 amplitude & $6.73(3.27)$ & $5.82(2.95)$ & 0.150 & 0.29 \\
\hline S2 amplitude & $2(1.31)$ & $2.46(1.3)$ & 0.073 & 0.35 \\
\hline N100 ratio & $0.36(0.31)$ & $0.51(0.34)$ & $0.017^{*}$ & 0.46 \\
\hline N100 difference & $4.73(3.35)$ & $3.36(2.62)$ & $0.030^{*}$ & 0.46 \\
\hline
\end{tabular}

UHR, ultra-high-risk group; FEP, first-episode psychosis group. ERP, event-related potential. MMN, mismatch negativity.

aSome subjects failed to stay before the ERP session was terminated. The number of analyzable P50/N100 subjects was 20 UHR and 13 FEP. The number of analyzable MMN subjects was 18 UHR and 16 FEP.

${ }^{b}$ Chi-square tests.

${ }^{*} p<0.05$.

${ }^{* *} p<0.01$. 
group's smaller amplitude of MMN was not so evident when pairwise comparisons were made between UHR and controls as well as between FEP and controls. Similarly, when UHR and FEP were compared to the control group separately, no significant difference could be found in P50 parameters. The only significant differences remained in N100-related parameters: the FEP had a significant lower amplitude in N100 S1 amplitude compared to the controls, and the UHR had a higher N100 S2 amplitude than the controls, while the larger N100 amplitude ratio became insignificant in both groups, but the N100 difference remained significantly smaller in the FEP group but not in the UHR group. Comparisons between control subjects and patients in P50 ratios, N100 differences and MMN values are shown in Figure 1. The average MMN waveforms are demonstrated in Figure 2.

TABLE 2 | Demographics and ERP results of three groups (SD in parentheses). ${ }^{a}$

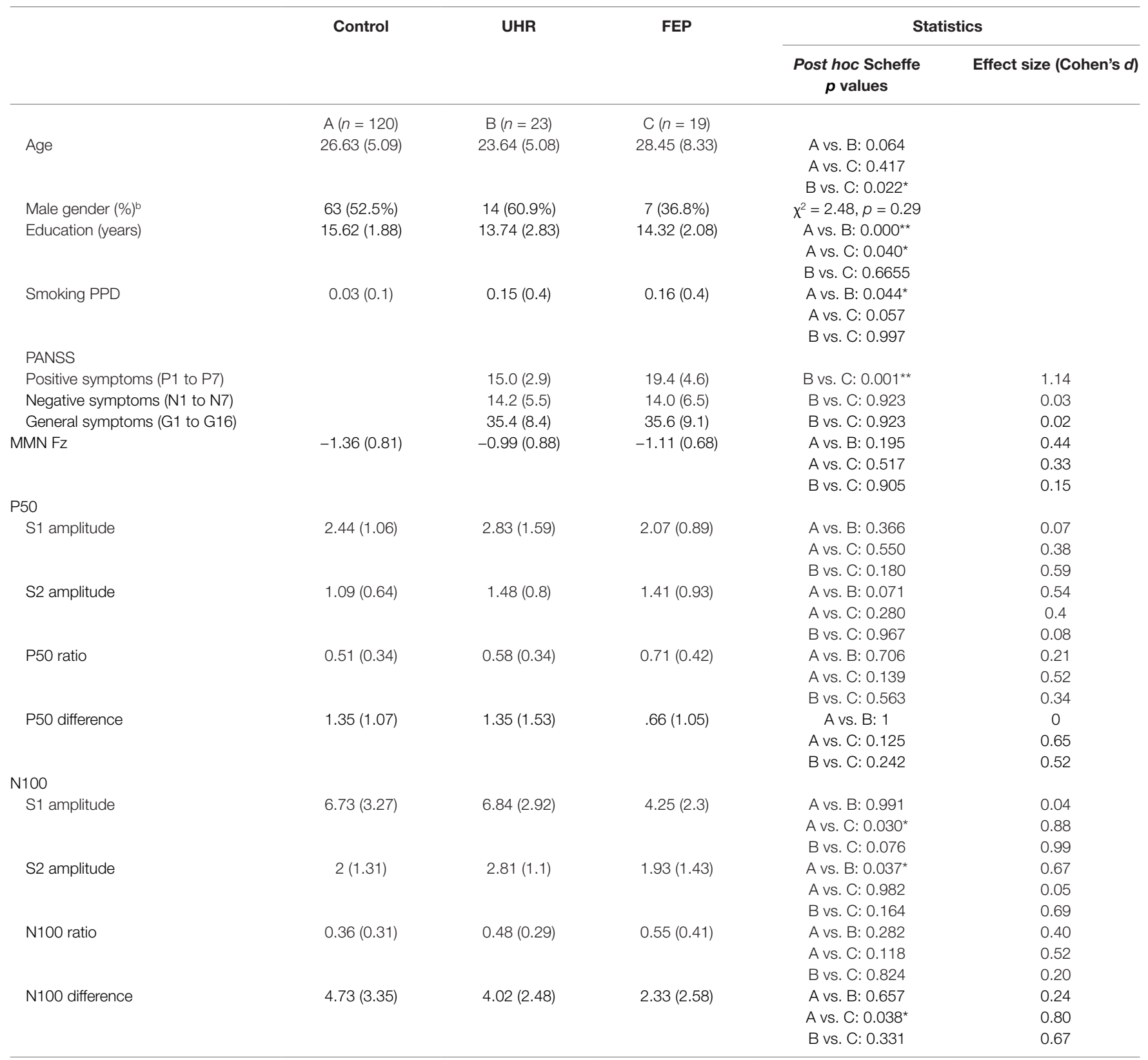

UHR, ultra-high-risk group; FEP, first-episode psychosis group.

aSome subjects failed to stay before the ERP session was terminated. The number of analyzable P50/N100 subjects was 20 UHR and 13 FEP. The number of analyzable MMN subjects was 18 UHR and 16 FEP.

${ }^{b}$ Chi-square tests.

${ }^{*} p<0.05$.

${ }^{* *} p<0.01$. 
(A)

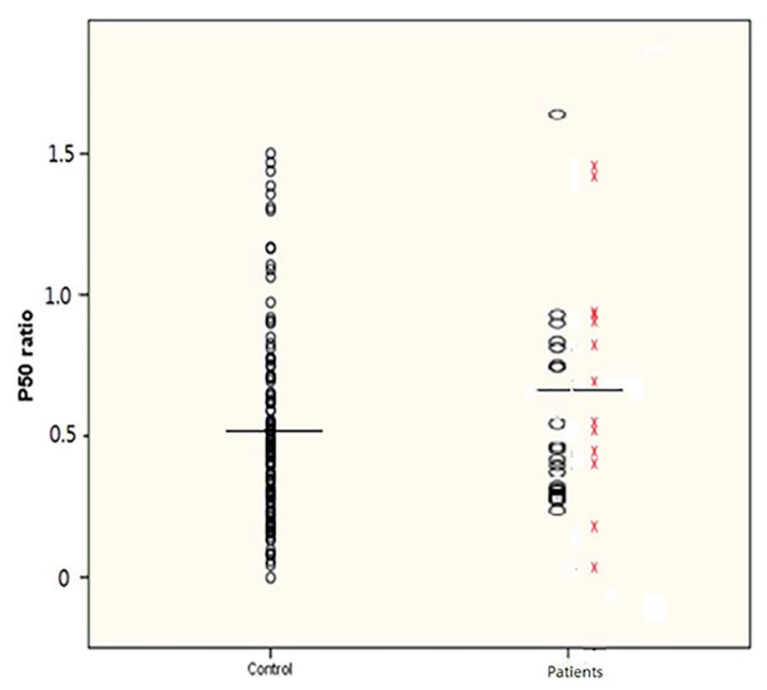

(B)

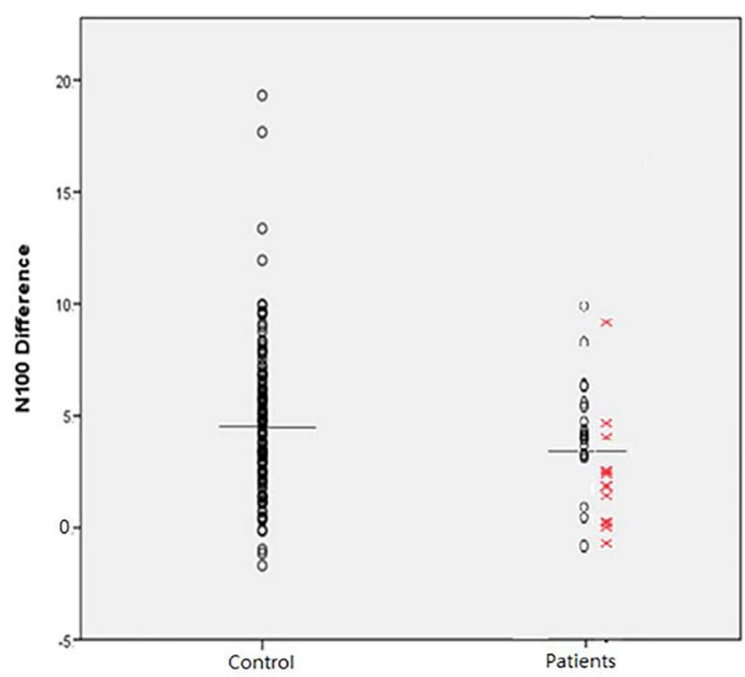

(C)

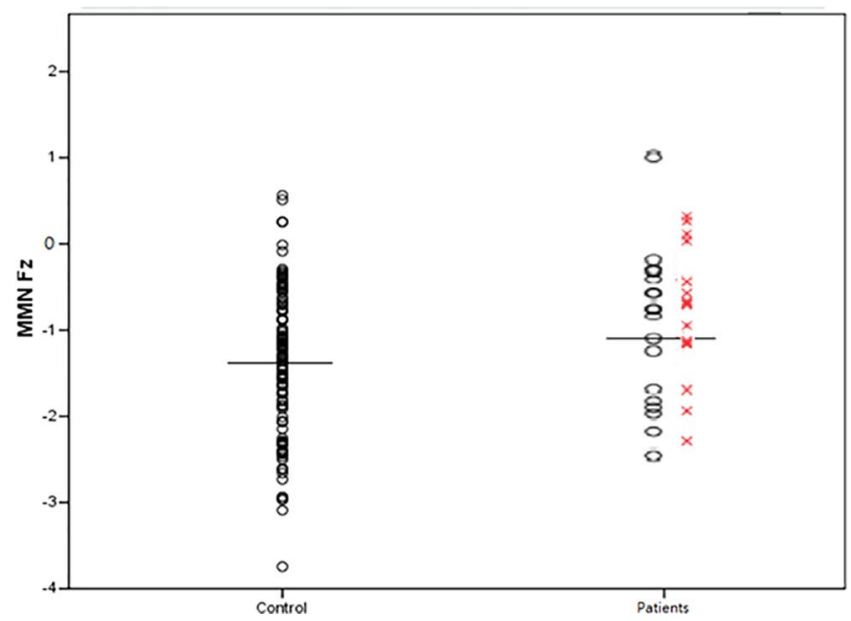

FIGURE 1 | P50 ratios (S2 amplitude/S1 amplitude) (A), N100 differences ( $\mu$ V; S2 amplitude - S1 amplitude) (B), and mismatch negativity (MMN) at electrode Fz (C) of individual participants between groups. The horizontal lines indicate the mean values within control vs. patient group, while the patient group consists of ultra-high-risk (UHR; oval) and first-episode psychosis (FEP; X) subjects. For P50 and N100, a larger ratio (S2/S1) and a smaller difference (S1 - S2) indicate poorer sensory gating. For MMN, a larger (less negative) value indicates poor deviance detection.

A head-to-head comparison of ERP results between the drugnaive subjects and the antipsychotic-short-exposure subjects is shown in Table 3. Apparently, there was no significant difference in any P50/N100 and MMN parameter between these two groups.

\section{DISCUSSION}

It is believed that clinical and cognitive deficits of psychosis may be due to dysfunction at the earlier stages of information processing (56). Bora and Murray's meta-analysis highlighted that cognitive deficits are already established before the prodromal phases of psychosis (57), compatible with our previous publication regarding neurocognitive performance in different stages of preand early-psychotic states (58). Such neurocognitive disturbance might represent different components of auditory modality in sensory processing dysfunctions in schizophrenia, and our neurophysiological paradigms measuring "pre-attentive, passive" auditory ERPs in UHR and first-episode psychosis subjects can add valuable information to this field (59).

Although many studies of MMN were conducted on subjects with UHR states, only few have also measured P50/N100 in the same study (60). Also, several studies have included patients with first-episode psychosis and examined them separately from chronic schizophrenia, and most publications reported 


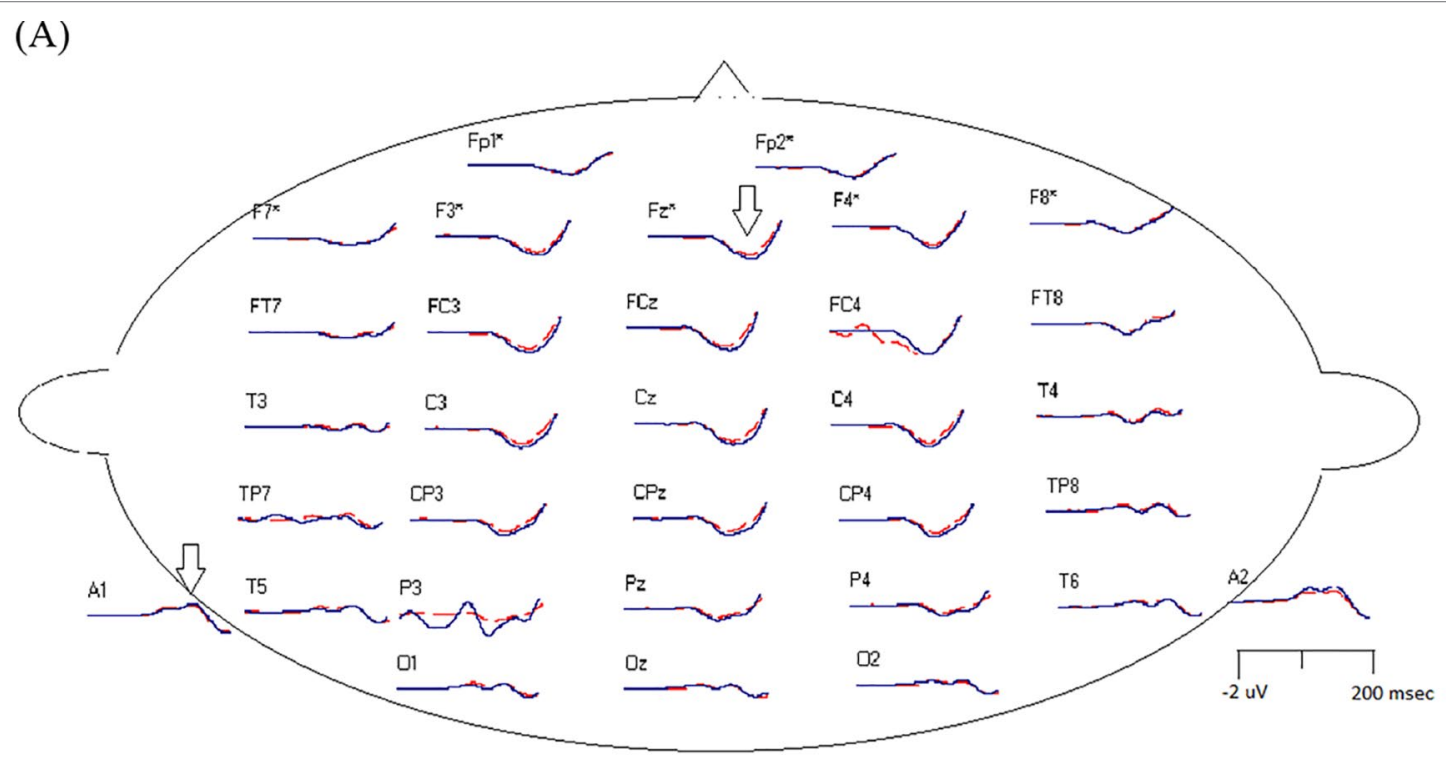

(B)

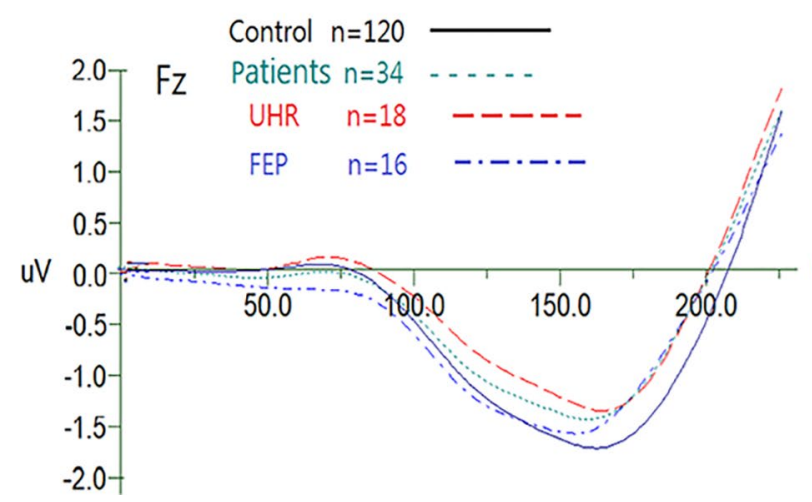

(C)

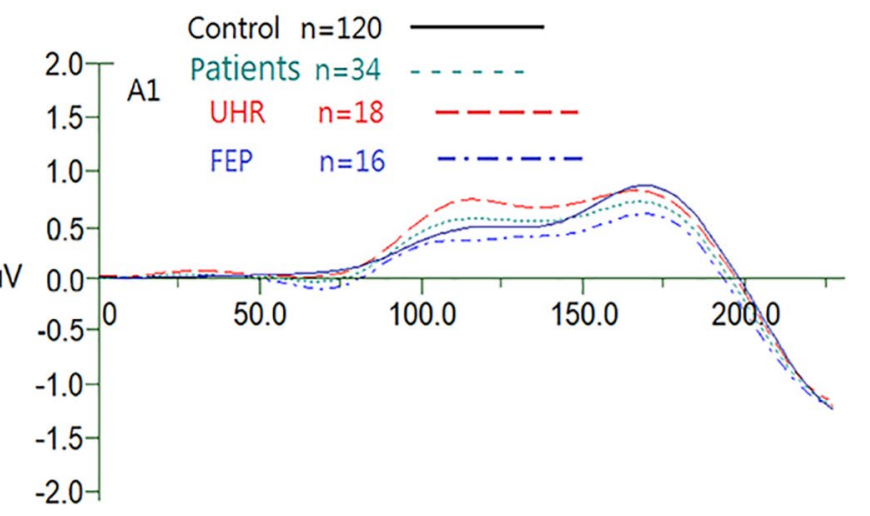

FIGURE 2 | (A) Demonstrated grand average MMN waveforms for healthy control subjects (in blue) and patients (in red). The arrows indicated the waveform reversed in polarity at the mastoid electrodes, which is typical for MMN. A close-up of grand average waveform at electrode Fz and $\mathrm{A} 1$ (mastoid) electrodes is shown in (B) and (C).

auditory pre-attentive (passive) ERPs after the patients had been treated with antipsychotics. For example, Koshiyama et al. investigated duration vs. frequency MMN in 14 FEP patients, 16 UHR individuals, and 16 healthy controls. They concluded that duration $\mathrm{MMN}$ is superior to frequency $\mathrm{MMN}$ as a trait marker in the early stages of psychosis, and a smaller duration MMN amplitude in early stages of psychosis may reflect altered developmental process rather than progressive brain pathology (61). However, most of their patients with either FEP or UHR have been treated with antipsychotic medication prior to the experiment, leaving a possible confounder in their interpretation of results.

As Haigh et al's meta-analysis of MMN in first-episode schizophrenia patients highlighted a need to conduct study on medication-naive individuals (26), our report is one of the few studies focused on P50/N100/duration MMN in drug-naive or minimally treated FEP and UHR patients. Consistent with our previous report when drug naivety was not strictly defined in that study population, a linear trend of more deviance from controls across different levels of clinical severity was noticed in $\mathrm{P} 50$ ratios (S2/S1) and N100 differences, even though the differences in P50 and N100 between control and clinical groups were not statistically significant (39). Specific to study on sensory gating adopting P50/N100 paradigms, our findings are in line with Shaikh et al.s 36 unmedicated patients who met attenuated psychosis syndrome (equivalent to our UHR) and have already exhibited P50 sensory gating deficits at this pre-psychotic state (62). Similarly, Brockhaus-Dumke et al. found impaired P50 suppression (S2/S1 ratio) in all clinical severities (at risk, true prodromal, first episode, and chronic 
TABLE 3 | ERP results of control/drug-naive/antipsychotic-short-exposure groups (SD in parentheses). ${ }^{a}$

\begin{tabular}{|c|c|c|c|c|c|}
\hline & \multirow[t]{2}{*}{ Control $(n=120)$} & \multirow[t]{2}{*}{ Drug-naive $(n=21)$} & \multirow{2}{*}{$\begin{array}{l}\text { Antipsychotic short } \\
\text { exposure }(n=21)\end{array}$} & \multicolumn{2}{|c|}{ Statistics } \\
\hline & & & & $\begin{array}{c}\text { Post hoc Scheffe } \\
p \text { values }\end{array}$ & $\begin{array}{l}\text { Effect size } \\
\text { (Cohen's } d \text { ) }\end{array}$ \\
\hline & $A$ & $\mathrm{~B}$ & $\mathrm{C}$ & & \\
\hline \multirow[t]{3}{*}{ MMN Fz } & $-1.36(0.81)$ & $-1.08(0.88)$ & $-1.00(0.67)$ & A vs. B: 0.396 & 0.34 \\
\hline & & & & A vs. C: 0.267 & 0.45 \\
\hline & & & & B vs. C: 0.967 & 0.10 \\
\hline \multicolumn{6}{|l|}{ P50 } \\
\hline \multirow[t]{3}{*}{ S1 amplitude } & $2.44(1.06)$ & $2.77(1.77)$ & $2.33(1.00)$ & A vs. B: 0.572 & 0.29 \\
\hline & & & & A vs. C: 0.936 & 0.10 \\
\hline & & & & B vs. C: 0.551 & 0.31 \\
\hline \multirow[t]{3}{*}{ S2 amplitude ${ }^{b}$} & 1.09 (0.64) & $1.32(0.56)$ & $1.56(1.02)$ & A vs. B: 0.480 & 0.36 \\
\hline & & & & A vs. C: $0.027^{b}$ & 0.67 \\
\hline & & & & B vs. C: 0.600 & 0.28 \\
\hline \multirow[t]{3}{*}{ P50 ratio } & $0.51(0.34)$ & $0.54(0.24)$ & $0.71(0.45)$ & A vs. B: 0.942 & 0.09 \\
\hline & & & & A vs. C: 0.083 & 0.56 \\
\hline & & & & B vs. C: 0.402 & 0.46 \\
\hline \multirow[t]{3}{*}{ P50 difference } & $1.35(1.07)$ & $1.45(1.51)$ & $0.77(1.23)$ & A vs. B: 0.947 & 0.09 \\
\hline & & & & A vs. C: 0.141 & 0.53 \\
\hline & & & & B vs. C: 0.239 & 0.50 \\
\hline \multicolumn{6}{|l|}{ N100 } \\
\hline \multirow[t]{3}{*}{ S1 amplitude } & $6.73(3.27)$ & $6.06(3.01)$ & $5.61(2.96)$ & A vs. B: 0.751 & 0.21 \\
\hline & & & & A vs. C: 0.391 & 0.35 \\
\hline & & & & B vs. C: 0.923 & 0.15 \\
\hline \multirow[t]{3}{*}{ S2 amplitude } & $2(1.31)$ & $2.64(0.92)$ & $2.32(1.56)$ & A vs. B: 0.209 & 0.50 \\
\hline & & & & A vs. C: 0.631 & 0.24 \\
\hline & & & & B vs. C: 0.783 & 0.24 \\
\hline \multirow[t]{3}{*}{ N100 ratio } & $0.36(0.31)$ & $0.50(0.21)$ & $0.52(0.42)$ & A vs. B: 0.270 & 0.47 \\
\hline & & & & A vs. C: 0.149 & 0.49 \\
\hline & & & & B vs. C: 0.989 & 0.06 \\
\hline \multirow{3}{*}{ N100 difference } & $4.73(3.35)$ & $3.43(2.40)$ & $3.30(2.86)$ & A vs. B: 0.336 & 0.40 \\
\hline & & & & A vs. C: 0.214 & 0.43 \\
\hline & & & & B vs. C: 0.993 & 0.05 \\
\hline
\end{tabular}

aSome subjects failed to stay before the ERP session was terminated. The number of analyzable P50/N100 subjects was 15 for drug-naive and 18 for antipsychotic short exposure. The number of analyzable MMN subjects was 18 for drug-naive and 16 for antipsychotic short exposure.

${ }^{b}$ Post hoc Scheffe test revealed significant differences between control and antipsychotic-short-exposure groups $(p=0.027)$.

schizophrenia), while impaired N100 suppression (S1 - S2 difference) was also seen in all clinical groups except in the at-risk subjects (63); the latter is exactly the same with our finding. Specific to studies on MMN, our results are similar to Mondragon-Maya et al.s (23) and During et al.'s (64) MMN and P3a studies, which revealed no impaired deviance detection ability among antipsychotic naive first-episode psychosis patients and individuals at clinical high risk for psychosis and control subjects.

In addition to verifying previous studies, we took a closer look into our findings. When UHR and FEP were compared to normal controls separately, the directions of changes of ERP parameters are of great interest. Based on the sensory gating failure theory, the patients are expected to reveal smaller S1 and larger S2 in P50 signals. This pattern could only be seen in our FEP subjects but not the UHR patients, while the latter exhibited larger, but not smaller, S1, together with larger S2. Although none of these findings reached statistical significance, our findings derived from subjects not confounded by antipsychotic medication might give a hint to understand the dynamic changes of sensory gating in patients with schizophrenia during the progress of their illness. Also, even though no difference in MMN could be detected when UHR and FEP were compared to normal controls separately, collectively as a patient group, their MMN deviance detection ability is lower than that of normal controls, also a finding not confounded by antipsychotics.

Two major limitations of the current study are worth noting. The relatively small sample size of the UHR $(n=$ $23)$ and FEP $(n=19)$ groups limits our statistical power to detect smaller differences between groups, such as dividing the pure drug-naive UHR and FEP from those who had short exposure to antipsychotics in either group, but we believe that the majority of our participants had limited impact by antipsychotic treatment, comprising a very valuable sample. Future studies recruiting a larger sample would be necessary to verify our findings. Second, none of our UHR patients converted to full-blown psychosis during a period of 4 weeks. We did not know how many of them would eventually develop psychosis after 1 or 2 years, while previous studies suggested that ERP performance of the converters were likely worse than that of the nonconverters $(11,13,65)$.

In summary, our ERP results of antipsychotic-free subjects with UHR state and first-episode psychosis are 
not much different from those studies that did not control antipsychotic medication status. Our drug-naive subjects showed no significant difference from their antipsychoticshort-exposure counterparts as well. If this is true, it will be convenient to use this modality to measure patient's sensory gating performance regardless of the impact of antipsychotics, at least at the pre- and early-psychotic states. Nonetheless, we will examine if there are differences in ERP performance between baseline and by the end of a 4-week exposure to antipsychotic treatment. A longer follow-up of prospective longitudinal study will be helpful to provide more evidence to elucidate the role of antipsychotic medication on an individual's neurophysiological performance at different stages of psychosis.

\section{ETHICS STATEMENT}

This study was carried out in accordance with the recommendations of the guidelines of National Taiwan University Hospital Research Ethics Committee with written informed consent from all subjects. All subjects gave written informed consent, including written assent given by minors with informed consent from their parents

\section{REFERENCES}

1. Keshavan MS, Tandon R, Boutros NN, Nasrallah HA. Schizophrenia, "just the facts": what we know in 2008 Part 3: neurobiology. Schizophr Res (2008) 106(2-3):89-107. doi: 10.1016/j.schres.2008.07.020

2. Blow N. Neuroscience tools: brain insights. Nat Methods (2008) 5(11):981-7. doi: 10.1038/nmeth1108-981

3. Javitt DC, Spencer KM, Thaker GK, Winterer G, Hajos M. Neurophysiological biomarkers for drug development in schizophrenia. Nat Rev Drug Discov (2008) 7(1):68-83. doi: 10.1038/nrd2463

4. Lieberman JA, Perkins D, Belger A, Chakos M, Jarskog F, Boteva K, et al. The early stages of schizophrenia: speculations on pathogenesis, pathophysiology, and therapeutic approaches. Biol Psychiatry (2001) 50(11):884-97. doi: 10.1016/S0006-3223(01)01303-8

5. Rissling AJ, Light GA. Neurophysiological measures of sensory registration, stimulus discrimination, and selection in schizophrenia patients. Curr Top Behav Neurosci (2010) 4:283-309. doi: 10.1007/7854_2010_59

6. Turetsky BI, Calkins ME, Light GA, Olincy A, Radant AD, Swerdlow NR. Neurophysiological endophenotypes of schizophrenia: the viability of selected candidate measures. Schizophr Bull (2007) 33(1):69-94. doi: 10.1093/schbul/ sbl060

7. Umbricht DS, Bates JA, Lieberman JA, Kane JM, Javitt DC. Electrophysiological indices of automatic and controlled auditory information processing in first-episode, recent-onset and chronic schizophrenia. Biol Psychiatry (2006) 59(8):762-72. doi: 10.1016/j.biopsych. 2005.08.030

8. Turetsky BI, Greenwood TA, Olincy A, Radant AD, Braff DL, Cadenhead KS, et al. Abnormal auditory N100 amplitude: a heritable endophenotype in first-degree relatives of schizophrenia probands. Biol Psychiatry (2008) 64(12):1051-9. doi: 10.1016/j.biopsych.2008.06.018

9. Todd J, Michie PT, Schall U, Ward PB, Catts SV. Mismatch negativity $(\mathrm{MMN})$ reduction in schizophrenia-impaired prediction-error generation, estimation or salience? Int J Psychophysiol (2012) 83(2):222-31. doi: 10.1016/j.ijpsycho.2011.10.003

10. Perez VB, Woods SW, Roach BJ, Ford JM, McGlashan TH, Srihari VH, et al. Automatic auditory processing deficits in schizophrenia and clinical highrisk patients: forecasting psychosis risk with mismatch negativity. Biol Psychiatry (2014) 75(6):459-69. doi: 10.1016/j.biopsych.2013.07.038 in accordance with the Declaration of Helsinki. The protocol was approved by the Research Ethics Committee of National Taiwan University Hospital.

\section{AUTHOR CONTRIBUTIONS}

MHH and C-ML reviewed literature and designed this study. MHH and Y-TL did the ERP study and data analysis. Y-LC performed the statistics. C-ML oversaw the clinical trial. C-CL, T-JH and H-GH handled the early psychosis studies. All authors have helped to recruit subjects and involved in clinical and diagnostic assessments. MHH wrote the first draft of the manuscript. C-CL finalized the writing and editing of the manuscript. All authors contributed to and have approved the final manuscript.

\section{FUNDING}

This work was supported by the Ministry of Science and Technology (Grant Number MOST 103-2314-B-002-020-MY2, 106-2314-B-002-236-, and 107-2314-B-002-012-) and the National Science Council, Taiwan (Grant Numbers NSC 95-2221-E-002-028 and NSC 98-2314-B- 002-047-MY3).

11. Bodatsch M, Ruhrmann S, Wagner M, Muller R, Schultze-Lutter F, Frommann I, et al. Prediction of psychosis by mismatch negativity. Biol Psychiatry (2011) 69(10):959-66. doi: 10.1016/j.biopsych.2010.09.057

12. Erickson MA, Ruffle A, Gold JM. A meta-analysis of mismatch negativity in schizophrenia: from clinical risk to disease specificity and progression. Biol Psychiatry (2016) 79(12):980-7. doi: 10.1016/j.biopsych.2015.08.025

13. Bodatsch M, Brockhaus-Dumke A, Klosterkotter J, Ruhrmann S. Forecasting psychosis by event-related potentials-systematic review and specific meta-analysis. Biol Psychiatry (2015) 77(11):951-8. doi: 10.1016/j. biopsych.2014.09.025

14. Breier A. Diagnostic classification of the psychoses: historical context and implications for neurobiology. In: Charney DS Nestler EJ, editors. Neurobiology of mental illness. New York: Oxford University Press (2004). p. 237-46.

15. Mathalon DH, Ford JM, Rosenbloom M, Pfefferbaum A. P300 reduction and prolongation with illness duration in schizophrenia. Biol Psychiatry (2000) 47(5):413-27. doi: 10.1016/S0006-3223(99)00151-1

16. Premkumar P, Fannon D, Kuipers E, Cooke MA, Simmons A, Kumari V. Association between a longer duration of illness, age and lower frontal lobe grey matter volume in schizophrenia. Behav Brain Res (2008) 193(1):132-9. doi: 10.1016/j.bbr.2008.05.012

17. Tanskanen P, Ridler K, Murray GK, Haapea M, Veijola JM, Jaaskelainen E, et al. Morphometric brain abnormalities in schizophrenia in a populationbased sample: relationship to duration of illness. Schizophr Bull (2010) 36(4):766-77. doi: 10.1093/schbul/sbn141

18. Earls HA, Curran T, Mittal V. A meta-analytic review of auditory eventrelated potential components as endophenotypes for schizophrenia: perspectives from first-degree relatives. Schizophr Bull (2016) 42(6):1504-16. doi: 10.1093/schbul/sbw047

19. Hall MH, Rijsdijk F. Validating endophenotypes for schizophrenia using statistical modeling of twin data. Clin EEG Neurosci (2008) 39(2):78-81. doi: $10.1177 / 155005940803900211$

20. Magno E, Yeap S, Thakore JH, Garavan H, De Sanctis P, Foxe JJ. Are auditoryevoked frequency and duration mismatch negativity deficits endophenotypic for schizophrenia? High-density electrical mapping in clinically unaffected first-degree relatives and first-episode and chronic schizophrenia. Biol Psychiatry (2008) 64(5):385-91. doi: 10.1016/j.biopsych.2008.03.019 
21. Kaur M, Lagopoulos J, Lee RS, Ward PB, Naismith SL, Hickie IB, et al. Longitudinal associations between mismatch negativity and disability in early schizophrenia- and affective-spectrum disorders. Prog Neuropsychopharmacol Biol Psychiatry (2013) 46:161-9. doi: 10.1016/j. pnpbp.2013.07.002

22. Salisbury DF, Polizzotto NR, Nestor PG, Haigh SM, Koehler J, McCarley RW. Pitch and duration mismatch negativity and premorbid intellect in the first hospitalized schizophrenia spectrum. Schizophr Bull (2017) 43(2):407-16. doi: 10.1093/schbul/sbw074

23. Mondragon-Maya A, Solis-Vivanco R, Leon-Ortiz P, Rodriguez-Agudelo Y, Yanez-Tellez G, Bernal-Hernandez J, et al. Reduced P3a amplitudes in antipsychotic naive first-episode psychosis patients and individuals at clinical high-risk for psychosis. J Psychiatr Res (2013) 47(6):755-61. doi: 10.1016/j.jpsychires.2012.12.017

24. de Wilde OM, Bour LJ, Dingemans PM, Koelman JHTM, Linszen DH. Failure to find P50 suppression deficits in young first-episode patients with schizophrenia and clinically unaffected siblings. Schizophr Bull (2007) 33(6):1319-23. doi: 10.1093/schbul/sbm001

25. Bachmann S, Weisbrod M, Röhrig M, Schröder J, Thomas C, Scherg M, et al. MEG does not reveal impaired sensory gating in first-episode schizophrenia. Schizophr Res (2010) 121(1):131-8. doi: 10.1016/j.schres.2010.03.007

26. Haigh SM, Coffman BA, Salisbury DF. Mismatch negativity in first-episode schizophrenia: a meta-analysis. Clin EEG Neurosci (2017) 48(1):3-10. doi: $10.1177 / 1550059416645980$

27. Becker J, Gomes I, Ghisolfi ES, Schuch A, Ramos FL, Ehlers JA, et al. Clozapine, but not typical antipsychotics, correct P50 suppression deficit in patients with schizophrenia. Clin Neurophysiol (2004) 115(2):396-401. doi: 10.1016/j.clinph.2003.09.018

28. Umbricht D, Javitt D, Novak G, Bates J, Pollack S, Lieberman J, et al. Effects of clozapine on auditory event-related potentials in schizophrenia. Biol Psychiatry (1998) 44(8):716-25. doi: 10.1016/S0006-3223(97)00524-6

29. Korostenskaja M, Dapsys K, Siurkute A, Maciulis V, Ruksenas O, Kahkonen S. Effects of olanzapine on auditory P300 and mismatch negativity (MMN) in schizophrenia spectrum disorders. Prog Neuropsychopharmacol Biol Psychiatry (2005) 29(4):543-8. doi: 10.1016/j.pnpbp.2005.01.019

30. Light GA, Geyer MA, Clementz BA, Cadenhead KS, Braff DL. Normal P50 suppression in schizophrenia patients treated with atypical antipsychotic medications. AmJ Psychiatry (2000) 157(5):767-71. doi: 10.1176/appi.ajp.157.5.767

31. Adler LE, Olincy A, Cawthra EM, McRae KA, Harris JG, Nagamoto HT, et al. Varied effects of atypical neuroleptics on P50 auditory gating in schizophrenia patients. Am J Psychiatry (2004) 161(10):1822-8. doi: 10.1176/ appi.ajp.161.10.1822

32. Umbricht D, Javitt D, Novak G, Bates J, Pollack S, Lieberman J, et al. Effects of risperidone on auditory event-related potentials in schizophrenia. Int J Neuropsychopharmacol (1999) 2(4):299-304. doi: 10.1017/ S1461145799001595

33. Fusar-Poli P, Bonoldi I, Yung AR, Borgwardt S, Kempton MJ, Valmaggia L, et al. Predicting psychosis: meta-analysis of transition outcomes in individuals at high clinical risk. Arch Gen Psychiatry (2012) 69(3):220-9. doi: 10.1001/ archgenpsychiatry.2011.1472

34. Fusar-Poli P, Borgwardt S, Bechdolf A, Addington J, Riecher-Rossler A, Schultze-Lutter F, et al. The psychosis high-risk state: a comprehensive state-of-the-art review. JAMA Psychiatry (2013) 70(1):107-20. doi: 10.1001/ jamapsychiatry.2013.269

35. McGorry PD, Yung AR, Phillips LJ. The "close-in" or ultra high-risk model: a safe and effective strategy for research and clinical intervention in prepsychotic mental disorder. Schizophr Bull (2003) 29(4):771-90. doi: 10.1093/oxfordjournals.schbul.a007046

36. Tsuang MT, Van Os J, Tandon R, Barch DM, Bustillo J, Gaebel W, et al. Attenuated psychosis syndrome in DSM-5. Schizophr Res (2013) 150(1):315. doi: 10.1016/j.schres.2013.05.004

37. Keshavan MS, Delisi LE, Seidman LJ. Early and broadly defined psychosis risk mental states. Schizophr Res (2011) 126(1-3):1-10. doi: 10.1016/j. schres.2010.10.006

38. Liu CC, Lai MC, Liu CM, Chiu YN, Hsieh MH, Hwang TJ, et al. Follow-up of subjects with suspected pre-psychotic state in Taiwan. Schizophr Res (2011) 126(1-3):65-70. doi: 10.1016/j.schres.2010.10.028
39. Hsieh MH, Shan JC, Huang WL, Cheng WC, Chiu MJ, Jaw FS, et al. Auditory event-related potential of subjects with suspected pre-psychotic state and first-episode psychosis. Schizophr Res (2012) 140:243-49. doi: 10.1016/j. schres.2012.06.021

40. Liu CC, Chien YL, Hsieh MH, Hwang TJ, Hwu HG, Liu CM. Aripiprazole for drug-naive or antipsychotic-short-exposure subjects with ultra-high risk state and first-episode psychosis: an open-label study. J Clin Psychopharmacol (2013) 33(1):18-23. doi: 10.1097/JCP. 0b013e31827cb017

41. McGorry PD, Yung AR, Phillips LJ. The "close-in" or ultra high-risk model: a safe and effective strategy for research and clinical intervention in prepsychotic mental disorder. Schizophr Bull (2003) 29(4):771-90. doi: 10.1093/oxfordjournals.schbul.a007046

42. Lijffijt M, Moeller FG, Boutros NN, Burroughs S, Lane SD, Steinberg JL, et al. The role of age, gender, education, and intelligence in P50, N100, and P200 auditory sensory gating. J Psychophysiol (2009) 23(2):52-62. doi: 10.1027/0269-8803.23.2.52

43. Duncan CC, Barry RJ, Connolly JF, Fischer C, Michie PT, Naatanen $\mathrm{R}$, et al. Event-related potentials in clinical research: guidelines for eliciting, recording, and quantifying mismatch negativity, P300, and N400. Clin Neurophysiol (2009) 120(11):1883-908. doi: 10.1016/j. clinph.2009.07.045

44. Shan JC, Hsieh MH, Liu CM, Chiu MJ, Jaw FS, Hwu HG. More evidence to support the role of S2 in P50 studies. Schizophr Res (2010) 122(1-3):270-2. doi: $10.1016 /$ j.schres.2010.05.026

45. Light GA, Williams LE, Minow F, Sprock J, Rissling A, Sharp R, et al. Electroencephalography (EEG) and event-related potentials (ERPs) with human participants. Curr Protoc Neurosci (2010); 52(1):6.25.1-24. doi: 10.1002/0471142301.ns0625s52

46. Olincy A, Martin L. Diminished suppression of the P50 auditory evoked potential in bipolar disorder subjects with a history of psychosis. Am J Psychiatry (2005) 162:43-9. doi: 10.1176/appi.ajp.162.1.43

47. Clementz BA, Geyer MA, Braff DL. Multiple site evaluation of P50 suppression among schizophrenia and normal comparison subjects. Schizophr Res (1998) 30(1):71-80. doi: 10.1016/S0920-9964(97)00122-9

48. de Wilde OM, Bour LJ, Dingemans PM, Koelman JHTM, Linszen DH. A meta-analysis of P50 studies in patients with schizophrenia and relatives: differences in methodology between research groups. Schizophr Res (2007) 97(1-3):137-51. doi: 10.1016/j.schres.2007.04.028

49. Shan JC, Liu CM, Chiu MJ, Liu CC, Chien YL, Hwang TJ, et al. A diagnostic model incorporating p50 sensory gating and neuropsychological tests for schizophrenia. PLoS One (2013) 8(2):e57197. doi: 10.1371/journal. pone.0057197

50. Boutros NN. Lack of blinding in gating studies. Schizophr Res (2008) 103(13):336. doi: 10.1016/j.schres.2008.02.017

51. Semlitsch HV, Anderer P, Schuster P, Presslich O. A solution for reliable and valid reduction of ocular artifacts, applied to the P300 ERP. Psychophysiology (1986) 23(6):695-703. doi: 10.1111/j.1469-8986.1986. tb00696.x

52. Nagamoto HT, Adler LE, Waldo MC, Freedman R. Sensory gating in schizophrenics and normal controls: effects of changing stimulation interval. Biol Psychiatry (1989) 25:549-61. doi: 10.1016/ 0006-3223(89)90215-1

53. Light GA, Swerdlow NR, Thomas ML, Calkins ME, Green MF, Greenwood $\mathrm{TA}$, et al. Validation of mismatch negativity and P3a for use in multi-site studies of schizophrenia: characterization of demographic, clinical, cognitive, and functional correlates in COGS-2. Schizophr Res (2015) 163(1-3):63-72. doi: 10.1016/j.schres.2014.09.042

54. Kiang M, Braff DL, Sprock J, Light GA. The relationship between preattentive sensory processing deficits and age in schizophrenia patients. Clin Neurophysiol (2009) 120(11):1949-57. doi: 10.1016/j.clinph.2009.08.019

55. Lin YT, Liu CM, Chiu MJ, Liu CC, Chien YL, Hwang TJ, et al. Differentiation of schizophrenia patients from healthy subjects by mismatch negativity and neuropsychological tests. PLoS One (2012) 7(4):e34454. doi: 10.1317/ journal.pone. 0034454

56. Horvath S, Mirnics K. Breaking the gene barrier in schizophrenia. Nat Med (2009) 15(5):488-90. doi: 10.1038/nm0509-488 
57. Bora E, Murray RM. Meta-analysis of cognitive deficits in ultra-high risk to psychosis and first-episode psychosis: do the cognitive deficits progress over, or after, the onset of psychosis? Schizophr Bull (2014) 40(4):744-55. doi: $10.1093 /$ schbul/sbt085

58. Liu CC, Hua MS, Hwang TJ, Chiu CY, Liu CM, Hsieh MH, et al. Neurocognitive functioning of subjects with putative pre-psychotic states and early psychosis. Schizophr Res (2015) 164(1-3):40-6. doi: 10.1016/j.schres.2015.03.006

59. Braff DL, Light GA. Preattentional and attentional cognitive deficits as targets for treating schizophrenia. Psychopharmacology (Berl) (2004) 174(1):75-85. doi: 10.1007/s00213-004-1848-0

60. Lepock JR, Mizrahi R, Korostil M, Bagby RM, Pang EW, Kiang M. Eventrelated potentials in the clinical high-risk (CHR) state for psychosis: a systematic review. Clin EEG Neurosci (2018) 49(4):215-25. doi: $10.1177 / 1550059418755212$

61. Koshiyama D, Kirihara K, Tada M, Nagai T, Koike S, Suga M, et al. Duration and frequency mismatch negativity shows no progressive reduction in early stages of psychosis. Schizophr Res (2017) 190:32-8. doi: 10.1016/j.schres.2017.03.015

62. Shaikh M, Dutt A, Broome MR, Vozmediano AG, Ranlund S, Diez A, et al. Sensory gating deficits in the attenuated psychosis syndrome. Schizophr Res (2015) 161(2-3):277-82. doi: 10.1016/j.schres.2014.12.021

63. Brockhaus-Dumke A, Schultze-Lutter F, Mueller R, Tendolkar I, Bechdolf A, Pukrop R, et al. Sensory gating in schizophrenia: P50 and N100 gating in antipsychotic-free subjects at risk, first-episode, and chronic patients. Biol Psychiatry (2008) 64(5):376-84. doi: 10.1016/j.biopsych.2008.02.006

64. During S, Glenthoj BY, Oranje B. Effects of blocking D2/D3 receptors on mismatch negativity and P3a amplitude of initially antipsychotic naive, first episode schizophrenia patients. Int J Neuropsychopharmacol (2015) 19(3):pyv109. doi: 10.1093/ijnp/pyv109

65. Shaikh M, Valmaggia L, Broome MR, Dutt A, Lappin J, Day F, et al. Reduced mismatch negativity predates the onset of psychosis. Schizophr Res (2012) 134(1):42-8. doi: 10.1016/j.schres.2011.09.022

Conflict of Interest Statement: The authors declare that the research was conducted in the absence of any commercial or financial relationships that could be construed as a potential conflict of interest.

Copyright (c) 2019 Hsieh, Lin, Chien, Hwang, Hwu, Liu and Liu. This is an openaccess article distributed under the terms of the Creative Commons Attribution License (CC BY). The use, distribution or reproduction in other forums is permitted, provided the original author(s) and the copyright owner(s) are credited and that the original publication in this journal is cited, in accordance with accepted academic practice. No use, distribution or reproduction is permitted which does not comply with these terms. 